\title{
Measurement of Chili Pepper Plants Size Based on Mathematical Morphology
}

\author{
Yun Gao, Xiaoyu Li, Kun Qi, and Hong Chen \\ College of Engineering, Huazhong Agricultural University \\ Wuhan, China \\ angelclouder@qq. com
}

\begin{abstract}
Since chili pepper plant size directly reflects the state of plant growth, a method for pepper measurement of plants size was discussed here. Pepper plants were shot from above once per week in the greenhouse since being field planted in spring. The method of processing the pepper plant images was studied, in which the image segmentation of combination of color space and the image morphological operations were applied. And the major axis and minor axis of pepper plant, for describing the size of the plant, were calculated from single connected component in the image being processed. According to the method, a program for pepper plant size measurement based on MATLAB was developed. Experimental results have demonstrated that the method is more reasonable and accurate than artificial measure.
\end{abstract}

Keywords: pepper plant; size measurement; segmentation; morphological operation; major axis and minor axis.

\section{Introduction}

Chili pepper, which plays an important role in the year-round vegetable supply in china, is an important commercial-orientated crop in the country ${ }^{[1,2]}$. During the cultivation of chili pepper plants, the growth state and morphological directly influences the suitability of a plant for cultivation, its overall yield and its economic coefficient $^{[3]}$. The time of each growth phase, the number of leaves, weight of fresh leaf, leaf area, thickness of leaf, size of leaf, and so on are used to describe the growth state. However, the size of plants, as the intuitive and important factor to describe the growth stage and growth state, has less been studied, because of the difficult measurement. As comparing the differences in size between the same capsicum species does help research on capsicum cultivation techniques and improve the yield and quality of pepper. In this work, we developed a method to detect the size of capsicum plants using computer vision technology.

The chili pepper plants were photographed in the greenhouse for the size measurement method developed. An algorithm, using image segmentation method to separate pepper plant from the background, and image binarization method to make the image black and white, after that, the morphology method was utilized to make single frame pepper plant image into a single connected graph. Finally, the longest diameter 
and the shortest diameter, as plant morphology parameters, of the single connected graph, were introduced. Experiments verified that the algorithm was effective, with comparing measurement data with the tape to data calculated by the algorithm.

\section{Image Acquisition}

50 chili pepper plants, which were planted in the spring in the greenhouse of Hubei Academy of Agricultural Science and Technology, photos have been taken for study, by using single $\mu 300$ Olympus digital camera and a tripod metal photographic PTZ. Chili pepper seedlings were transplanted from the seedbed to the greenhouses, as planting spacing of $40 \mathrm{~cm}$ and seedling spacing of $45 \mathrm{~cm}$. One week later, chili pepper plants were photographed once a week for seven weeks. 150 pictures were collected each time, and three pictures were taken from one chili pepper plant.

In the photo collection, the camera was placed on the tripod metal photographic PTZ, just perpendicular to the plant, and shot the plant from above, as shown in Figure 1 , in which $\mathrm{H}$ is the distance from the camera lens to the ground. Between three times shooting, the camera was rotated 120 degrees in the horizontal direction. The image resolution is $1024 \times 768$, each image is saved as a JPEG file. To improve the adaptability of the analysis method, the shooting was not under extra lighting but natural light. The first three weeks after the beginning of image acquisition, each image contained only one plant. From the fourth week, chili pepper plants grew staggered, and not suitable for image acquisition. So the study object in this paper is the images acquired from the shooting of the first three weeks. Fig. 1 shows how the pictures have been taken, in which $\mathrm{H}$ is the vertical distance from the actual shooting of the camera lens to the ground pepper cultivation.

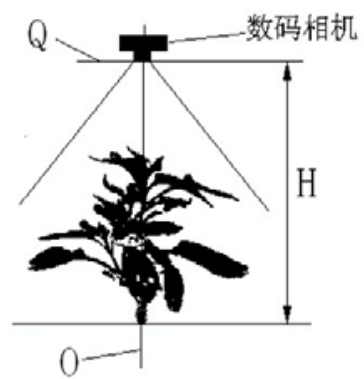

拍摄俯视图

Fig. 1. Sketch map for shooting method

\section{Image Segmentation}

\subsection{RGB Color Image Segmentation}

To detect the size of chili pepper plants, pepper images need to be segmented from background. From pepper picture in Fig. 2, we can see the background of pepper 
plants mainly compos of the soil covered with plastic film, the black section on the upper right corner of the image is irrigation tube road under the plastic film. As the main color of pepper plants is green, some parts of the leaves and petioles are yellow and the color is very close to the background color of the soil and the film, which makes the image segmentation very difficult.

At present, there are two main methods to use the color characteristics for the color image segmentation: one is changes the two-dimensional color images into grayscale images, and grayscale threshold segmentation algorithm is used for gray image segmentation; another is based on color segmentation, and in the color space it directly limits each RGB value of the color space and separates the chili pepper plant and the background $^{[4,5]}$.

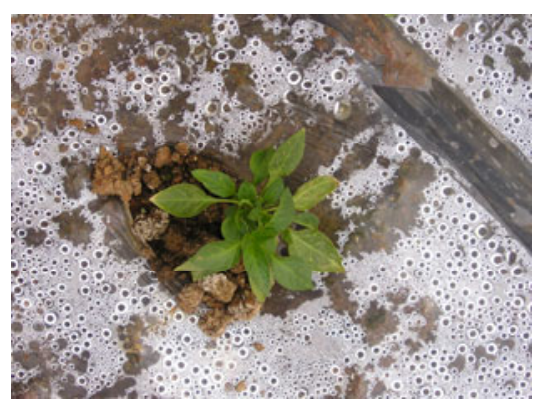

Fig. 2. Image of chili pepper plant

Major color spaces, used in color region segmentation today, are RGB color space and HIS color space. The images shown in Fig. 1, is segmented for the Euclidean distance $^{[6]}$. The Euclidean distance between $z$ and $m$ is given by

$$
D(\mathrm{z}, \mathrm{m})=\|\mathrm{z}-\mathrm{m}\|=\left[(\mathrm{z}-\mathrm{m})^{T}(\mathrm{z}-\mathrm{m})\right]^{1 / 2}=\left[\left(z_{R}-m_{R}\right)^{2}+\left(z_{G}-m_{G}\right)^{2}+\left(z_{B}-m_{B}\right)^{2}\right]^{1 / 2}
$$

Wherein $m$ stands for the RGB column vector of average color from the region of chili pepper plant to be segmented and $z$ stands for an arbitrary point in RGB space. $\|$.$\| is the norm of the argument, and subscripts R, G$ and $B$, stands for the RGB values of vectors $m$ and $z$. Figs.3 (a)through (d) show the segmentation results with $\mathrm{T}=25$, 45,60 and $m=[96.0202126 .0374$ 45.5014]'. Here $m$ is a vector of mean RGB values in the plant region.

In Figs.3 (a)through (d) show when $\mathrm{T}$ is too small, the deterioration of plant appears in (a). when $\mathrm{T}$ is too large, the background cannot be segmented well from the image .

To directly set the threshold of $\mathrm{RGB}$ values with $\mathrm{R}(\mathrm{i}, \mathrm{l})<=140 \& \mathrm{R}(\mathrm{i}, \mathrm{l})>=$ $59 \& \mathrm{~B}(\mathrm{i}, 1)<=105 \& \mathrm{~B}(\mathrm{i}, 1)>=3 \& \mathrm{G}(\mathrm{i}, 1)<=195 \& \mathrm{G}(\mathrm{i}, 1)>=92$ can not have an well segmentation result, which shows in Fig. 4.

In the study we found the yellow soil could be segmented well in HIS color space by using the threshold algorithm, but the plastic film and irrigation tube road couldn't. The image processing result was shown in Figure 5 with $\mathrm{H}(\mathrm{i}, \mathrm{l})<=1 \& \mathrm{H}(\mathrm{i}, \mathrm{l})>0.15$ $\& \mathrm{I}(\mathrm{i}, 1)<0.55$. 


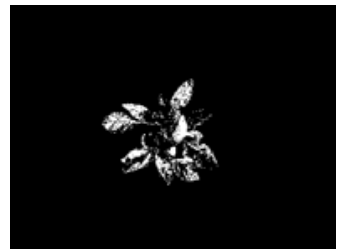

(a) $\mathrm{T}=25$

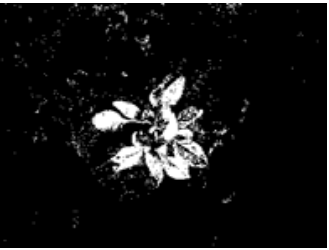

(b) $\mathrm{T}=45$

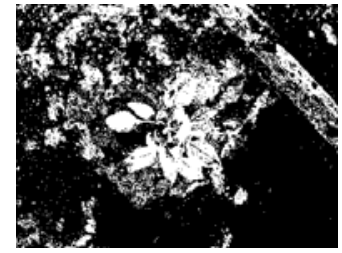

(c) $\mathrm{T}=60$

Fig. 3. Segmentation with $\mathrm{T}=25,45,60$, respectively

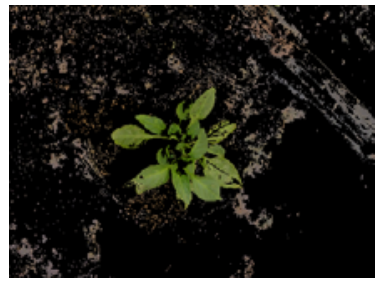

Fig. 4. Segmentation in RGB color space

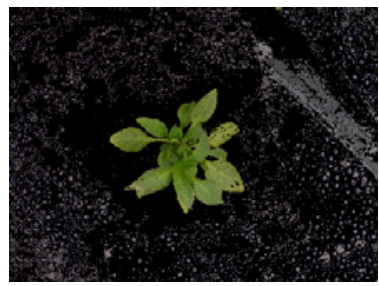

Fig. 5. Segmentation in HIS color space

When we used the Euclidean distance method in RGB color space on Fig.5 for segmentation by using the option 'euclidean' with $\mathrm{T}=50$, and available results was shown in Fig. 6. From the figure, the plant's incompleteness is reduced and the background is almost removed.

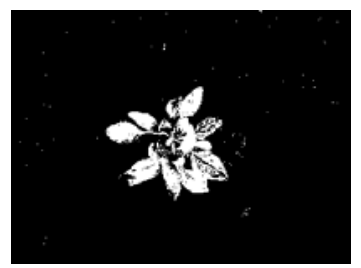

Fig. 6. Segmentation using the threshold algorithm in HIS color space and the option 'euclidean' with $\mathrm{T}=50$ 


\subsection{Combination of Color Space Image Segmentation}

As in our previous studies, we found it is difficult to achieve good results for segmenting pepper images in only one color space. So the algorithm combining the segmentation in RGB color space and the segmentation in HIS color space was used here. In two spaces setting threshold of each color vector for several tests we have $\mathrm{H}$ $(\mathrm{i}, \mathrm{l})<=1 \& \mathrm{H}(\mathrm{i}, \mathrm{l})>0.15 \& \mathrm{I}(\mathrm{i}, \mathrm{l})<0.55 \& \mathrm{R}(\mathrm{i}, \mathrm{l})<=0.543 \& \mathrm{R}(\mathrm{i}, \mathrm{l})>=0.231 \& \mathrm{~B}$ $(\mathrm{i}, \mathrm{l})<=0.333 \& \mathrm{~B}(\mathrm{i}, \mathrm{l})>=0 \& \mathrm{G}(\mathrm{i}, \mathrm{l})<=0.647 \& \mathrm{G}(\mathrm{i}, \mathrm{l})>=0.331$, and segmentation of Fig. 2 is shows in Fig.7.

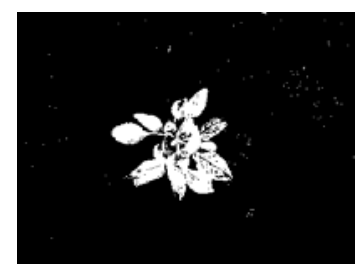

Fig. 7. Segmentation in combination of color space

Comparing with Fig.6, the background elimination of Fig.7 is similar, but the pepper plant in fig.7 in the division breakage is much better than that in Fig.6. Concerning the operation time of two methods, the preceding algorithm's operation time is $6.2970 \mathrm{~s}$ and the latter algorithm's operation time is $4.5160 \mathrm{~s}$. Compared to the preceding kind, the latter one saves time at approximately $28 \%$. If we proceed to process the image in fig. 7 using the wiener filter before it is segmented, it would reduce the division breakage of the plant, but the operation time would up to $8.5630 \mathrm{~s}$, the result image is shown in Fig.8.

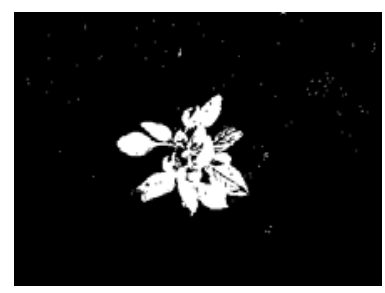

Fig. 8. Segmentation in combination of color space with wiener filter

\section{Image Morphological Operations}

\subsection{Opening and Closing}

After being operated by combination color space image segmentation, the image has some tiny noises in the background, and division breakages in plant's leaf surface and stems. We use morphological opening to remove the small noise in background. The 
morphological opening of $A$ by $B$, denoted $A \circ B$, is simply erosion of $A$ by $B$, followed by dilation of the result by $B^{[6]}$ :

$$
A \circ B=(A \Theta \mathrm{B}) \oplus \mathrm{B}
$$

The operation produced the result in Fig.9. The morphological opening algorithm can remove the thin protrusions and outward-pointing boundary irregularities. Here, the small isolated object in background were removed.

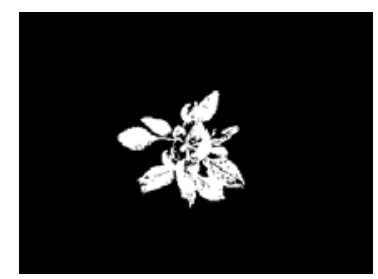

Fig. 9. Result of morphological opening

Morphological opening can fill the division breakage of the plant after being segmented. The morphological closing of $A$ by $B$, denoted $A \bullet B$, is a dilation followed by an erosion ${ }^{[6]}$ :

$$
A \bullet B=(A \oplus B) \Theta B
$$

Like opening, morphological closing tends to smooth the contours of objects. Unlike opening, however, it generally joins narrow breaks, fills long thin gulfs, and fills holes smaller than the structuring element. The following are results by using the structuring elements of $5 * 5$ and $10 * 10$ for closing operation on the image, which are shown in Fig. 10 (a) and (b).

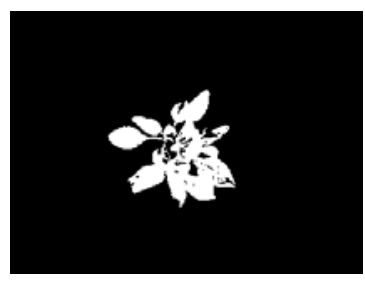

(a) Using the structuring elements of $5 * 5$

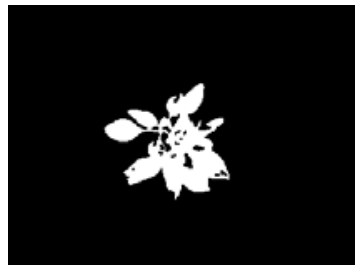

(b) Using the structuring elements of $10 * 10$

Fig. 10. Results of closing with structuring elements of $5 * 5$ and $10 * 10$, respectively

\subsection{One Connected Component}

To calculate the size of chili plants, the plant in Fig.10 needs to be transferred to a single connected component. In order to prevent chili plants becoming multiple connected components in one image, we applied labeling to process the image ${ }^{[7]}$. When 
there are more than one connected component in the image, morphological closing is repeatedly used to operate the image to fill the area between the breaking parts until the number of the connected component in the image becomes one. Comparison of results before and after operation are shown in Fig. 11.

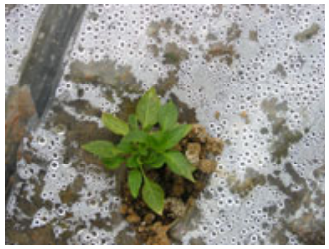

(a)original image

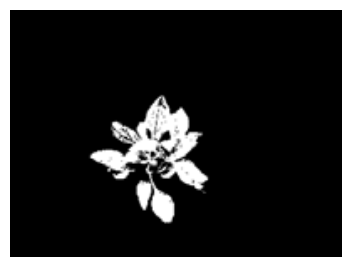

(b) Image using segmentation and morphological algorithm

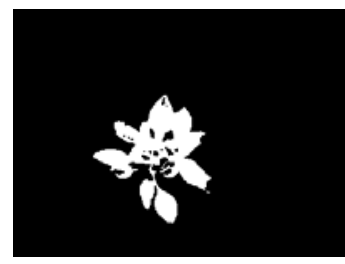

(c) One connected component

Fig. 11. One connected component processing

In Fig.11(a), the plant is segmented excessively and one under leaf separates from the main plant. When marking the image, there are two connected components. After being operated morphological closing twice, the image is shown in Fig.11(b), where the under leaf is connected to the main plant and the number of the connected components become one.

\subsection{Multiple Objects Processing}

When Chili pepper plants grow larger, the distance between each other becomes closer. In the third week of shooting, one image may not only have the main plant, but also have the other part of plant near to the main plant, which makes calculation of the plant size difficult. Therefore removing the object which contacts with the boundary of the image can make the connected components become one, as shown in Fig.12.

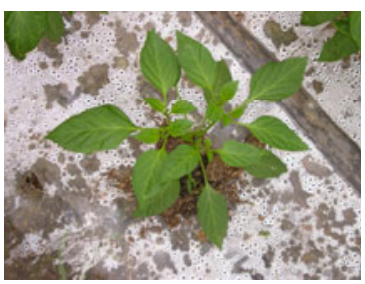

(a) original image

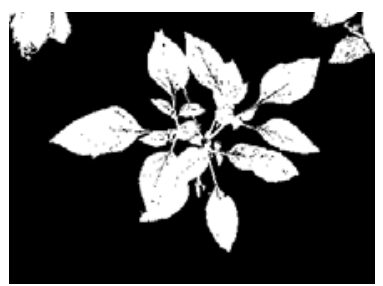

(b)image using segmentation and morphological algorithm

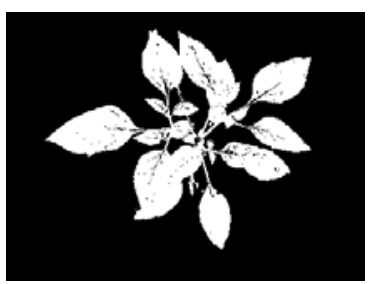

(c) removing the other plants

Fig. 12. Multiple objecst processing

In fig.12, we used the function "imclearborder" to eliminate the leaves from other plant near to the main plant which connect to image boundary, and reserved single connected phenomenon of the main plant ${ }^{[6]}$. 


\section{Detect the Size of the Pepper Plants}

\subsection{Major Axis and Minor Axis}

Diameter, major axis and minor axis are chili pepper plant size's simplest descriptors. The diameter of a boundary is defined as the Euclidean distance between the two farthest points on the boundary ${ }^{[8]}$. Its formula is

$$
\operatorname{Diam}(A)=\max _{i, j}\left[D\left(p_{i}, p_{j}\right)\right]
$$

Wherein $A$ stands for the region of one connected component of plant, $p_{i}, p_{j}$ stand for any two pixels in the plant region.

As the diameter is a useful descriptor, the major axis is defined as the line segment connecting a single pair of farthest points on the boundary of the plant. And the minor axis is defined as the line perpendicular to the major axis.

We use the lengths of major axis and minor axis as the descriptors for chili pepper plant size. When the image only includes a single connected component of the main chili pepper plant, function 'MajorAxis' and 'MinorAxis' are used to carry out the lengths of major axis and minor axis, According to the distance of shooting, the correction coefficient was used to make the lengths more precise. The equation is as follows:

$$
L_{r}=a \times L_{c}
$$

Wherein $a$ stands for the correction coefficient.

\subsection{Program of Measurement}

We developed programs to process images and calculate the sizes of chili pepper plants by using MATLAB's Graphical User Interface Development Environment. The surface of the software is shown in Fig.13 (a) and (b).

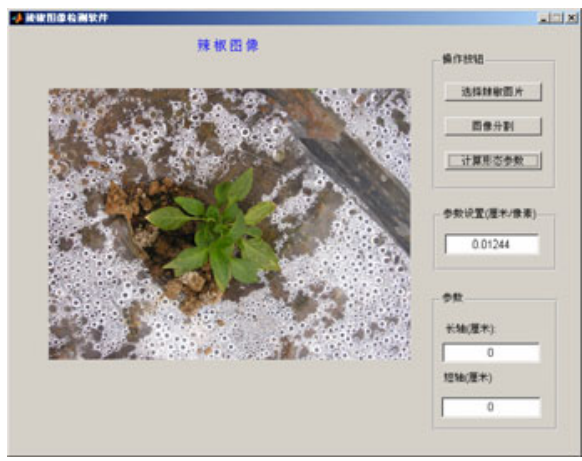

(a) Before processing

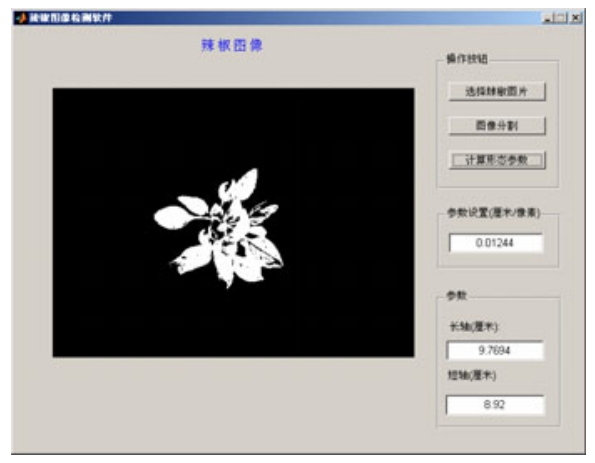

(b) After processing

Fig. 13. Surface of the software 
Fig.13 (a) is the surface before the plant image being processed, and fig.13(b) is the surface after the image being processed.

\subsection{Results Comparison}

The sizes of chili pepper plants measured in field compare to image measurement results. Manual measurement was conducted, including judging the direction of the major axis of the plant from above, using tape measure acquire the length of major axis along the direction and acquiring the length of minor axis along the direction perpendicular to the major axis.

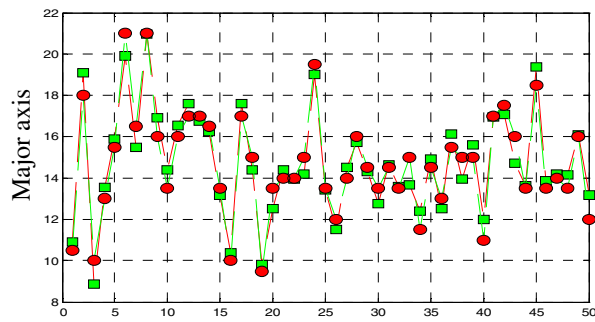

label of pepper plant

(a) major axis

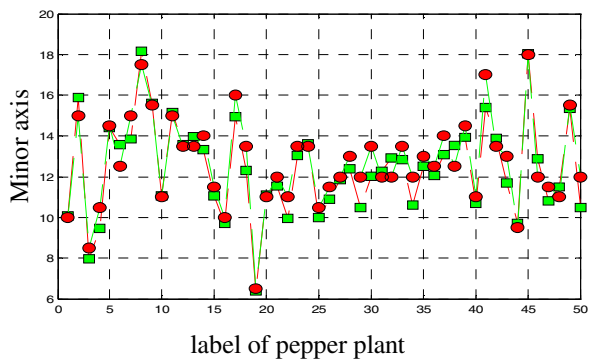

(b) minor axis

Fig. 14. Results Comparison

For example, 50 image acquired at the second week after transplant. Comparation of the results from manual measurement and the values from image measurements shows in Fig.14. And in Fig.14, (a)and(b) show two groups of major axis and minor axis values acquired through manual measurement and image measurement. Green squares stand for manual measurements, while red circles stand for image measurements.

From the figure, comparing the image measurements with the manual measurements, we can find the maximum error of 50 pairs of values is less than $1.3 \mathrm{~cm}$. In major axis values there are 35 pairs of points, $70 \%$ of all pairs of points, which have the error less than $0.7 \mathrm{~cm}$. Meanwhile in minor axis values there are 32 pairs of points have the error less than $0.7 \mathrm{~cm}$, a rate of $64 \%$.

The main reason for errors here is human vision. The direction of major axis in manual measurements couldn't be determined accurately. The other reason is because of precision of the tap measurement can only be up to $0.5 \mathrm{~cm}$. Meanwhile even the smallest wind would make the plant shaking during the field measures. In image measurements the difference between three measurements and their average is less than $0.5 \mathrm{~cm}$. Therefore, image measurement is more scientific and more accurate.

\section{Conclusion}

A method for chili pepper plants size measurement has been developed. Images were segmented in combination of RGB color space and HIS color space. And then 
the image morphological operations were applied to make the plant in one image so as to form a single connected component. And the major axis and minor axis of pepper plant, to describe the size of the plant, were calculated from single connected component in the image processed. According to the method, a program for pepper plant size measurement based on MATLAB was developed. Experimental results have demonstrated that the method is more reasonable and accurately than manual measure.

\section{References}

1. Reihardt, D., Kuhlemerier, C.: Plant architecture. EMBO Reports 3, 846-851 (2002)

2. Zou, X.: Cultivation Seasons and Cropping patterns of capsicum in china. Journal of China Capsicum 3, 32-35 (2002)

3. Gao, G., Pen, G.Z., Zhao, Z.: Development on the Expert System for Cultivation and Management of the Fresh Edible Capsicum in Guizhou. Guizhou Agricultural Sciences 5, 34-37 (2007)

4. Lin, K., Wu, J., Xu, L.: A Survey on Color Image Segmentation Techn iques. Journal of Image and Graphics 1, 1-10 (2005)

5. Ohta, Y., Kanade, T., Sakai, T.: Color information for region segmentation. Computer Graphics and Image Processing 3, 222-241 (1980)

6. Gonzalez, R.C., Woods, R.E.: Digital image processing, 2nd edn. (2007)

7. Wang, Z., Hu, R., Zhang, K.: Realization of Split-Merge Algorithm in Image Segmentation or Components Labeling. MINI- M ICROSYSTEMS 9, 1649-1651 (2004)

8. Liu, X., et al.: Fast Algorithm for Major andMinor Axes of Ra isins Image. Journal of Anhui Agri. 26, 11482-11483, 11509 (2008) 